\title{
SKEW POLYNOMIAL EXTENSIONS OF COMMUTATIVE NOETHERIAN JACOBSON RINGS
}

\author{
K. R. GOODEARL AND E. S. LETZTER
}

(Communicated by Lance W. Small)

\begin{abstract}
The Jacobson condition (i.e., that all prime ideals are semiprimitive) is proved to pass from a commutative noetherian ring $R$ to a skew polynomial ring $R[y ; \tau, \delta]$, assuming only that $\tau$ is an automorphism.
\end{abstract}

\section{INTRODUCTION}

This note is concerned with the prime ideal structure of a skew polynomial ring $S=R[y ; \tau, \delta]$ over a noetherian ring $R$ with respect to an automorphism $\tau$ and a (left) $\tau$-derivation $\delta$ (cf. [7]). An unanswered question in this setting is whether $S$ must satisfy the Jacobson condition (i.e., every prime ideal is an intersection of primitive ideals) when $R$ satisfies the same property. Some positive answers are known even for non-noetherian coefficient rings: Watters [15] proved that $K[y]$ is Jacobson for any Jacobson ring $K$, and Irving [9] showed that an iterated skew polynomial extension $T$ of a commutative Jacobson ring $K$ is Jacobson if $K$ is central in $T$ (see also [12]). On the other hand, examples have been constructed of non-noetherian commutative Jacobson rings $K$ with skew polynomial extensions $K[y ; \tau, \delta]$ that are not Jacobson; see Pearson and Stephenson [14] for an example in which $\delta=0$, and see Bergen, Montgomery, and Passman [1] or Ferrero and Kishimoto [3] for examples in which $\tau=1$. Within the noetherian context, affirmative answers to the problem were given by Goldie and Michler [4] when $\delta$ is trivial, and by Jordan [10] when $\tau$ is the identity.

The aim of this note is to provide an affirmative answer to the above question when $R$ is commutative noetherian but no restrictions are placed upon $\tau$ or $\delta$. Such a result has remained unavailable despite the thorough analyses of the commutative case by Irving [8] and the first author [5]. Our methods rely in part on the techniques introduced in [6] as well as on the results in [5]. Moreover, it is not assumed that $R$ be filtered, graded, or affine.

We impose the blanket hypotheses throughout that $R$ is a commutative noetherian ring, that $S=R[y ; \tau, \delta]$, and that $\tau$ is an automorphism of $R$, However, commutativity of $R$ is not needed for (2.2) and (3.1).

Received by the editors October 18, 1993.

1991 Mathematics Subject Classification. Primary 16D30, 16P40, 16 S36.

The research of the first author was partially supported by a National Science Foundation grant. 


\section{INDUCED VS. NONINDUCED PRIME IDEALS}

Throughout this section we let $P$ denote an arbitrary prime ideal of $S$. If $A$ is a ring and $I$ is an ideal of $A$, then $N(I)$ denotes the intersection of all the prime ideals containing $I$ and $J(I)$ the intersection of all the right primitive ideals containing $I$. The reader is referred to $[7,13]$ for further explanations of undefined terms.

2.1. By $[6,5.3,5.5]$, we may fix a prime ideal $Q$ of $R$, minimal over $P \cap R$, that satisfies the following property: If $A$ denotes the Goldie quotient ring of $R / Q$, then $P$ is the right annihilator in $S$ of a nonzero $A$-S-bimodule factor $M$ of $A \otimes_{R} S$ such that $M_{S / P}$ is torsionfree. Next, set $U=S / Q S$, and let $e$ denote the coset $1+Q S$. Observe that we may identify ${ }_{R} U_{S}$ with $(R / Q) \otimes_{R} S$ by an isomorphism that sends $e$ to $1 \otimes 1$, and under this identification we may view ${ }_{(R / Q)} U$ as a free left $(R / Q)$-module with basis

$$
\left\{1 \otimes 1,1 \otimes y, 1 \otimes y^{2}, \ldots\right\} .
$$

Also, observe that as a left $R$-module, $A \otimes_{R} S$ is isomorphic to an Ore localization of ${ }_{R} U$.

It follows from the above choice of $Q$ that ann $U_{S} \subseteq P$, since ann $U_{S}=$ ann $\left(A \otimes_{R} S\right)_{S}$. Our analysis divides into the two cases determined by whether or not $P=$ ann $U_{S}$, and we begin with an incomparabilty result.

2.2. Lemma. (Here $R$ need not be commutative.) Suppose that $J$ is an ideal of $S$ properly containing $P$. If $P \neq$ ann $U_{S}$, then $J \cap R \nsubseteq Q$.

Proof. By [6, 4.6], the set $\mathscr{C}$ of regular elements of $R /(P \cap R)$ forms an Ore set (of regular elements of $S / P$ ) in both $R /(P \cap R)$ and $S / P$, and the ring $E=(R /(P \cap R)) \mathscr{C}^{-1}$ is artinian. Letting $F=(S / P) \mathscr{C}^{-1}$, we see that the canonical embedding of $R /(P \cap R)$ into $S / P$ extends uniquely to an embedding of $E$ into $F$. Now choose an ideal $I$ of $S$ that contains $P$ and is maximal among those ideals of $S$ whose intersection with $R$ lies within $Q$. Standard arguments reveal that $I$ is a prime ideal of $S$ disjoint from $\mathscr{C}$. Consequently, if $I$ strictly contains $P$, then $I$ extends to a proper nonzero ideal of $F$ (e.g., $[7,9.22])$. Next, it follows from $[6,5.7,5.8]$ that $F_{E}$ is finitely generated when $P \neq$ ann $U_{S}$. However, if $F$ has finite length as a right $E$-module, then $F$ is a simple artinian ring. Therefore, $I=P$ and the lemma follows.

\subsection{Lemma. $(P+Q S) \cap R=Q$.}

Proof. We may assume without loss of generality that $P \cap R \neq Q$, and it therefore follows from the minimality of $Q$ that $P \cap R$ is not prime. Moreover, it suffices to prove that $(P+Q S) \cap R \subseteq Q$. Next, by [5, 3.1], either $P \cap R$ is semiprime or $R /(P \cap R)$ has a unique associated prime. We first consider the case where $R /(P \cap R)$ is semiprime, and we let $Q, Q_{2}, \ldots, Q_{n}$ be the distinct prime ideals of $R$ minimal over $P \cap R$. Note that $n \geq 2$ and $Q_{n} Q_{n-1} \cdots Q_{2} Q \subseteq$ $P \cap R$. Hence,

$$
Q_{n} Q_{n-1} \cdots Q_{2}[(P+Q S) \cap R] \subseteq P \cap R \subseteq Q .
$$

Since $Q_{n} Q_{n-1} \cdots Q_{2} \nsubseteq Q$, it follows that $(P+Q S) \cap R \subseteq Q$ in this case. 
Now assume that $R /(P \cap R)$ has a unique associated prime. Consequently, $Q$ is the unique prime ideal of $R$ minimal over $P \cap R$ and $\mathscr{C}_{R}(Q) \subseteq \mathscr{C}_{R}(P \cap R)$. Therefore, $\mathscr{C}_{R}(Q) \subseteq \mathscr{C}_{S}(P)$ by $[6,4.6]$. Hence, if there exists an element $c \in$ $(P+Q S) \cap(R \backslash Q)$, then $c \in \mathscr{C}_{S}(P)$. Next observe that there exists a positive integer $n$ such that $Q^{n} \subseteq P \cap R$ while $Q^{n-1} \nsubseteq P \cap R$. However, it now follows that $Q^{n-1} c \subseteq Q^{n-1}(P+Q S) \subseteq P$, in contradiction to the regularity of $c$ modulo $P$. Therefore, $(P+Q S) \cap R \subseteq Q$ and the lemma follows.

The proof of the following proposition is adapted from [4, 10].

2.4. Proposition. If $P \neq \operatorname{ann} U_{S}$ and $Q$ is semiprimitive, then $P$ is semiprimitive.

Proof. For $t=0,1, \ldots$ set

$$
\begin{aligned}
K_{t} & =\left\{a \in R \mid e .\left(a y^{t}+a_{t-1} y^{t-1}+\cdots+a_{0}\right) \in U P \text { for some } a_{0}, \ldots, a_{t-1} \in R\right\} \\
& =\left\{a \in R \mid a y^{t}+a_{t-1} y^{t-1}+\cdots+a_{0} \in P+Q S \text { for some } a_{0}, \ldots, a_{t-1} \in R\right\} .
\end{aligned}
$$

Then let $K=K_{n}$, where $n$ is the minimum value for $t$ such that

$$
0 \neq e .\left(a_{t} y^{t}+a_{t-1} y^{t-1}+\cdots+a_{0}\right) \in U P
$$

for some $a_{0}, \ldots, a_{t} \in R$. (The existence of $n$ follows from the assumption that $P \neq$ ann $U_{S}$.) Note, since $\tau$ is an automorphism, that $K$ is an ideal of $R$ containing $Q$, and observe, for $a \in K$, that $a \notin Q$ if and only if $0 \neq e \cdot\left(a y^{n}+a_{n-1} y^{n-1}+\cdots+a_{0}\right) \in U P$ for some $a_{0}, \ldots, a_{n-1} \in R$. In particular, $K$ properly contains $Q$. Moreover, since $(P+Q S) \cap R \subseteq Q$ by (2.3), it follows that $n \geq 1$.

Now let $M$ be a maximal ideal of $R$ that contains $Q$. We claim that either $J(P) \cap R \subseteq M$ or $K \subseteq M$. To prove this claim, assume that $J(P) \cap R \nsubseteq M$. Choose $j \in J(P) \cap R$ such that $j \notin M$. There then exist $m \in M$ and $b \in R$ such that $1=m+j b$. Since $j b \in J(P)$, there exists a polynomial $f=$ $c y^{\ell}+c_{\ell-1} y^{\ell-1}+\cdots+c_{0} \in S$, with $c, c_{0}, \ldots, c_{\ell-1} \in R$ and $c \neq 0$, such that $(1-j b) f=m f \equiv 1(\bmod P)$. Hence, $e \cdot m f \equiv e(\bmod U P)$. Now choose $a \in K \backslash Q$. There then exists a polynomial $p=a y^{n}+a_{n-1} y^{n-1}+\cdots+a_{0} \in S$, with $a_{0}, \ldots, a_{n-1} \in R$, for which $0 \neq e . p \in U P$. Assume for the moment that $\ell \geq n$, and observe that

$$
a f-p \tau^{-n}(c) y^{\ell-n}
$$

has degree less than $\ell$. It now follows from a straightforward induction that $e . a^{k} f \equiv e . r(\bmod U P)$ for some nonnegative integer $k$ and some polynomial $r \in S$ with degree $d<n$. Hence, we have

$$
e . a^{k} m f=m \cdot e \cdot a^{k} f \equiv m . e . r=e \cdot m r \quad(\bmod U P),
$$

and since $a^{k} m f \equiv a^{k}(\bmod P)$, we see that $e \cdot a^{k} \equiv e \cdot m r \quad(\bmod U P)$. Consequently, $e .\left(a^{k}-m r\right) \in U P$. However, $a^{k}-m r$ has degree strictly less than $n$. Therefore, it follows from the choice of $n$ that $e .\left(a^{k}-m r\right)=0$. Hence, $a^{k}-m r_{0} \in Q$, where $r_{0}$ is the constant term of $r$. But this last statement implies that $a^{k} \in M$, because $Q \subseteq M$. Thus $a \in M$, and it therefore follows from the choice of $a$ that $K \subseteq \bar{M}$. This verifies the claim. Furthermore, it follows from the claim that $J(P) \cap K \subseteq M$. Because $M$ was an arbitrary maximal ideal of $R$ containing $Q$, we now see that $J(P) \cap K \subseteq J(Q)=Q$. 
But this inclusion means that $J(P) \cap R \subseteq Q$, since $K \nsubseteq Q$. Thus by (2.2), $J(P)=P$, and the lemma is proved.

2.5. Lemma. Assume that $P=\operatorname{ann} U_{S}$. Then $P \cap R$ is $(\tau, \delta)$-prime, and $P=(P \cap R) S=S(P \cap R)$. Consequently, if $\tau$ and $\delta$ also denote their induced actions on $R /(P \cap R)$, and $y$ also denotes its image in $S / P$, then $S / P=$ $(R /(P \cap R))[y ; \tau, \delta]$.

Proof. Set $I=P \cap R$. It follows from [6, 5.9ii] that there exists an $n \in \mathbb{N}$ such that $\tau^{n}(Q)=Q$ and such that $\left\{Q, \tau(Q), \ldots, \tau^{n-1}(Q)\right\}$ is the set of prime ideals of $R$ minimal over $P \cap R$. In particular, $N(I)$ is $\tau$-stable. Now suppose that $I=Q$. Then $I$ is $\tau$-stable and therefore $(\tau, \delta)$-stable (e.g., [6, 2.1v]). Hence, $I S=S I$, and $P=\operatorname{ann}(S / I S)_{S}=I S$. Further, it is a triviality that $I$ is $(\tau, \delta)$-prime. Next, assume that $I \neq Q$. Consequently, $I$ is not a prime ideal, and so $I$ is a $(\tau, \delta)$-prime ideal by $[5,3.1]$. It therefore follows from $[5,3.3]$ that $P_{0}=I S=S I$ is a prime ideal of $S$. Moreover, $P_{0} \subseteq P$ and $P_{0} \cap R=P \cap R=I$.

Because $Q$ is minimal over $I$, and $R$ is commutative, it follows that $Q$ is an annihilator prime of $(R / I)_{R}$. In particular, $Q$ is an annihilator prime of $\left(S / P_{0}\right)_{R}$. Hence, by [6, 5.5], $P_{0} \supseteq$ ann $U_{S}=P$. The lemma follows.

2.6. Lemma. Suppose that $Q$ is a maximal ideal of $R$ and that $S / P$ is artinian. Then $S / P$ has finite length as a right $R$-module.

Proof. First, it follows from [6,4.4] that every prime ideal of $R$ minimal over $P \cap R$ is maximal, and so $R /(P \cap R)$ is artinian. Therefore, if $P \neq \operatorname{ann} U_{S}$, the desired conclusion follows from [6,5.9i]. Now assume that $P=\operatorname{ann} U_{S}$. Therefore, by (2.5), we may assume without loss of generality that $P=0$. But then $y$ is a regular noninvertible element of $S$, a contradiction to the fact that $S$ is artinian (e.g., $[13,3.1 .1])$.

\section{INDUCED BIMODULES}

Chapter 5 of [6] contains an extensive analysis of the prime ideals of $S$ that occur as annihilators of factors of bimodules of the form $A \otimes_{R} S$ where $A$ is the Goldie quotient ring of a prime factor ring of $R$. We shall need one element of the corresponding analysis of bimodule subfactors of $A \otimes_{R} S$, as follows. In the case of a bimodule factor, this lemma is a consequence of $[6,5.4,5.5]$.

3.1. Lemma. (Here $R$ need not be commutative.) Let $P$ be a prime ideal of $S$ and $Q$ a prime ideal of $R$, and let $A$ denote the Goldie quotient ring of $R / Q$. Further assume that $P$ is the right annihilator in $S$ of an $A$-S-bimodule subfactor $K$ of $A \otimes_{R} S$ that is torsionfree as a right $(S / P)$-module. Then every prime ideal in $R$ minimal over $P \cap R$ belongs to the $\tau$-orbit of $Q$.

Proof. Choose a nonzero element $\ell \in K$ and let $L=A . \ell . R$. It follows from $[6,4.6]$ that $R /(P \cap R)$ has an artinian quotient ring and that every regular element of $R /(P \cap R)$ is regular in $S / P$. Hence, $L$ is torsionfree as a right $(R /(P \cap R))$-module, and by Small's Theorem (e.g., [7, 10.10]) and [7, 6.3], it follows that every annihilator prime of $L_{R}$ is minimal over $P \cap R$. We leave to the reader the verification that $L$ has finite length as a left $A$-module. Now choose a simple $A$ - $R$-sub-bimodule $M$ of $L$. The right annihilator in $R$ of $M$ 
is a prime ideal, say $Q^{\prime}$, and we have just seen that $Q^{\prime}$ must be minimal over $P \cap R$. However, it follows from the proof in $[6,4.4]$ that $M$ is isomorphic to $A^{\tau^{j}}$ as an $A$ - $R$-bimodule, for some positive integer $j$. (As a left $A$-module, $A^{\tau^{j}}$ has the same structure as $A$, but the right $R$-module structure is defined by the operation $a * r=a \tau^{j}(r)$, for every $r \in R$ and $a \in A$.) It therefore follows that $Q^{\prime}=\tau^{-j}(Q)$, and the desired conclusion now follows from [6, 4.4].

\subsection{Proposition. Let $M$ be a maximal ideal of $R$. Then the right annihilator} in $S$ of $S / M S$ is prime.

Proof. Set $V=S / M S=(R / M) \otimes_{R} S$, and let $P$ denote a maximal annihilator prime of $V_{S}$. It follows from [6, 5.6iv] that $V$ is uniform as an $R$ - $S$-bimodule, and it is therefore easy to verify that every annihilator prime of $V_{S}$ is contained in $P$. If $P=$ ann $V_{S}$, then there is nothing to prove, and so we suppose otherwise. Next, let $0=V_{0} \subset V_{1} \subset \cdots \subset V_{n}=V$ be an affiliated series for $V$ (see, e.g., [7, p. 33]), where $n>1$, and set $P_{i}=\operatorname{ann}\left(V_{i} / V_{i-1}\right)_{s}$ for $1 \leq i \leq n$. (Note that $P_{1}=P$.) If $i>1$, it follows from [6, 5.6iii] that $V_{i} / \bar{V}_{i-1}$ has finite length as a left $R$-module. It therefore can be deduced from Lenagan's Theorem (e.g., $[7,7.10])$ that $\left(V_{i} / V_{i-1}\right)_{S}$ has finite length for $i>1$. However, it now follows from [7, 7.2] that $S / P_{i}$ is an artinian ring. In particular, each $V_{i} / V_{i-1}$ is torsionfree as a right $\left(S / P_{i}\right)$-module, and in view of (3.1), the prime ideals of $R$ minimal over $P \cap R$ are therefore maximal ideals. We may now conclude from (2.6) that each $S / P_{i}$ has finite length as a right $R$-module for $i>1$.

We next prove that $S / P=S / P_{1}$ is artinian and has finite length as a right $R$ module. If $P_{2}$ is an annihilator prime of $V_{S}$, then $P_{2} \subseteq P$ and there is nothing to prove. So we may assume otherwise. It then follows from [11, 1.2] that there is a series of links (e.g., [7, p. 178]) from $P_{2}$ to some annihilator prime $P^{\prime}$ of $V_{S}$. However, it now follows from [7, 7.2, 7.10] that $P^{\prime}$ is coartinian. Hence $P=P^{\prime}$ is coartinian, because $P^{\prime} \subseteq P$. Next, it follows from (3.1) that every prime ideal of $R$ minimal over $P \cap R$ is a maximal ideal. Thus $S / P$ has finite length as a right $R$-module by (2.6).

To conclude, it now follows that $V_{i} / V_{i-1}$ has finite length as a right $R$-module for all $1 \leq i \leq n$. But we are now forced to conclude that $V_{R}$ has finite length, an absurdity. The lemma follows.

\section{ASCENDANCY OF THE JACOBSON CONDITION}

4.1. Lemma. Assume that $R$ is artinian and $(\tau, \delta)$-prime. Then $S$ is a Jacobson ring.

Proof. First, it follows from [5, 2.3] and [4, 5*] that $R$ is $(\tau, \delta)$-simple. Also, $R$ is a Jacobson ring, and so by $[10,3.5]$ we may assume that $\tau$ is not the identity. Now assume that $R$ is $\tau$-prime. Then it follows from $[5,3.7]$ that $\delta$ is inner, and so the desired conclusion follows from $\left[4,1.11^{*}\right]$ and, for example, $[5,1.5 \mathrm{c}]$. It remains to consider the case that $R$ is not $\tau$-prime. Therefore, by $[5,2.6], R$ is $\delta$-prime and has a unique maximal ideal $M$. From [5, 2.6, 4.6] it follows that $S$ contains a subring $A=(R / M)\left[y^{\prime} ; \delta^{\prime}\right]$, where $y^{\prime} \in S$ and $\delta^{\prime}$ is a derivation of $R / M$, and it follows from [10,3.5] that $A$ is a Jacobson ring. It is proved in [5, 4.6] that $S$ is finitely generated as a left $A$-module. Therefore, $S$ is a Jacobson ring by [2, Theorem 1]. 
Recall that a prime ideal $P$ of $S$ is said to lie over a prime ideal $Q$ of $R$ when $Q$ is minimal over $P \cap R$.

4.2. Lemma. Assume that there exists a maximal ideal $M$ of $R$ such that the module $V=(S / M S)_{S}$ is faithful. Then $S$ is semiprimitive.

Proof. First suppose that $M$ is minimal. By (3.2), $S$ is prime, and so by [6, 5.12], the minimal prime ideals of $R$ are all contained within a single $\tau$-orbit. Therefore, all minimal prime ideals of $R$ are maximal, and so $R$ is artinian. Moreover, because $S$ is prime, and because nonzero $(\tau, \delta)$-ideals of $R$ induce to nonzero ideals of $S$, it follows that $R$ is $(\tau, \delta)$-prime. Hence, by $(4.1), S$ is semiprimitive. Thus we may assume that $M$ is not minimal.

Next, suppose that $\tau(M)=M$. Since $V_{S}$ is faithful, $M S$ cannot be an ideal of $S$, and so $M$ is not $\delta$-stable. Thus no ideal of $S$ contracts to $M$; see [6, 2.1v]. Now suppose that $N$ is a prime ideal of $S$ lying over $M$. From the preceding observation it follows that $N \cap R \neq M$, and so $I=N \cap R$ must be a $(\tau, \delta)$-prime ideal of $R$ by [5, 3.1]. Moreover, our assumption that $M$ not be a minimal prime ideal of $R$ guarantees that $I \neq 0$. Hence $I S$ is a nonzero ideal of $S$ contained in $M S$, a contradiction to the faithfulness of $V_{S}$. Thus, no prime ideal of $S$ lies over $M$. It therefore follows from [6, 5.7] that there exist no proper simple $R$ - $S$-bimodule factors of $V$, and so ${ }_{R} V_{S}$ is a simple bimodule. It is now straightforward to prove as follows that $S$ is right primitive: Let $K$ be a maximal right $S$-submodule of $V$, and let $J=\operatorname{ann}(V / K)_{S}$. Then $V J$ is an $R$-S-sub-bimodule of $V$ that is not equal to $V$. Hence $V J=0$, and so $J=0$ by the faithfulness of $V_{S}$. Therefore $V / K$ is a faithful simple right $S$-module.

Finally, assume that $\tau(M) \neq M$. Let $L=\bigcap_{i \in \mathbb{Z}} \tau^{i}(M)$, and note that $L$ is a semiprime, $\tau$-prime ideal. By $[5,3.1]$, for each $i \in \mathbb{Z}$ there exists a prime ideal of $S$ contracting to $\tau^{i}(M)$. Hence, there exists an ideal of $S$ contracting to $L$, and it follows, for example, from [6,2.1v] that $L$ is $(\tau, \delta)$-stable. Therefore, $L S=S L$ is an ideal of $S$ contained within $M S$, and so $L S=0$ because $V_{S}$ is faithful. Consequently, $L=0$, and hence $R$ is a semiprime, $\tau$-prime ring.

To conclude, let $J=J(S)$, and suppose that $J \neq 0$. Note that the set of leading coefficients of elements of $J$, together with 0 , namely the set

$$
\left\{a \in R \mid a y^{t}+a_{t-1} y^{t-1}+\cdots+a_{0} \in J \text { for some } a_{0}, \ldots, a_{t-1} \in R\right\},
$$

is a nonzero $\tau$-ideal of $R$. This ideal must contain a regular element since $R$ is $\tau$-prime, and therefore there exists a polynomial $f \in J$ with positive degree and regular leading coefficient. Since $1+f$ is a unit, there exists another polynomial $g$ such that $(1+f) g=1$. But the degree of $(1+f) g$ is certainly greater than zero, by the regularity of the leading coefficient of $f$, and we thus obtain a contradiction. Hence, $J=0$, and the lemma follows.

4.3. Theorem. Assume that $R$ is a commutative noetherian Jacobson ring. Then the skew polynomial ring $S=R[y ; \tau, \delta]$ is a Jacobson ring.

Proof. Suppose that the theorem is false, and let $P$ denote a maximally chosen nonsemiprimitive prime ideal of $S$. As in (2.1), we may select a prime ideal $Q$ of $R$ such that $Q$ is minimal over $P \cap R$ and such that $P$ is the annihilator in $S$ of an $A$ - $S$-bimodule factor of $A \otimes_{R} S$, where $A$ is the field of fractions for $R / Q$. If $P \neq \operatorname{ann}(S / Q S)_{S}$, then $P$ is semiprimitive, by (2.4). Therefore, 
by (2.5), we may assume without loss of generality that $P=0$. Furthermore, $Q$ is equal to the intersection of those maximal ideals of $R$ that contain it. In particular,

Therefore,

$$
Q S=\bigcap\{M S \mid M \in \max R \text { and } M \supseteq Q\} .
$$

$$
0=\operatorname{ann}(S / Q S)_{S}=\bigcap\left\{\operatorname{ann}(S / M S)_{S} \mid M \in \max R \text { and } M \supseteq Q\right\} .
$$

Next, it follows from the above equalities and (3.2) that if there exists no maximal ideal $M$ in $S$ such that $M \supseteq Q$ and $(S / M S)_{S}$ is faithful, then some intersection of nonzero prime ideals in $S$ is equal to zero, a contradiction to the fact that each nonzero prime ideal of $S$ is semiprimitive. Thus, there exists a maximal ideal $M$ in $R$ such that $(S / M S)_{S}$ is faithful. Therefore, it follows from (4.2) that $S$ is semiprimitive, a contradiction to our hypothesis. The theorem follows.

4.4. A question of Small. A possible generalization of the preceding theorem would include the replacement of the commutativity hypothesis with the assumption that $R$ satisfy a polynomial identity. L. W. Small has informed us of his unpublished proof that if $R$ is an affine PI algebra over a $(\tau, \delta)$ constant field $k$, then $S[u, v]=R[y ; \tau, \delta][u][v]$ is generically flat over $k[u]$, and consequently, $S$ is a Jacobson ring (cf. [13, 9.3.13]). Small further raises the following question: If $T$ is a filtered noetherian ring such that $\operatorname{gr} T$ is Jacobson, must $T$ also be Jacobson? (We thank L. W. Small for the remarks discussed here.)

\section{NOTE ADDED IN PROOF (DECEMBER 1994)}

A. D. Bell has communicated two counterexamples to Small's question; however, in one example the filtration is a $\mathbb{Z}$-filtration, while in the other, $\operatorname{gr} T$ is not noetherian. The following modification of Small's question remains open: If $T$ is a nonnegatively filtered noetherian ring such that $\operatorname{gr} T$ is Jacobson and noetherian, must $T$ be noetherian?

\section{REFERENCES}

1. J. Bergen, S. Montgomery, and D. S. Passman, Radicals of crossed products of enveloping algebras, Israel J. Math. 59 (1987), 167-184.

2. B. Cortzen and L. W. Small, Finite extensions of rings, Proc. Amer. Math. Soc. 103 (1988), 1058-1062.

3. M. Ferrero and K. Kishimoto, On differential rings and skew polynomials, Comm. Algebra 13 (1985), 285-304.

4. A. W. Goldie and G. O. Michler, Ore extensions and polycyclic group rings, J. London Math. Soc. (2) 9 (1974), 337-345.

5. K. R. Goodearl, Prime ideals in skew polynomial rings and quantized Weyl algebras, J. Algebra 150 (1992), 324-377.

6. K. R. Goodearl and E. S. Letzter, Prime ideals in skew and q-skew polynomial rings, Mem. Amer. Math. Soc. 109 (1994), 1-106.

7. K. R. Goodearl and R. B. Warfield, Jr., An introduction to noncommutative Noetherian rings, London Math. Soc. Stud. Texts, vol. 16, Cambridge Univ. Press, Cambridge, 1989.

8. R. S. Irving, Prime ideals of Ore extensions over commutative rings. II, J. Algebra 58 (1979), 399-423. 
9. $\ldots$, Generic flatness and the Nullstellensatz for Ore extensions, Comm. Algebra 7 (1979), 259-277.

10. D. A. Jordan, Noetherian Ore extensions and Jacobson rings, J. London Math. Soc. (2) 10 (1975), 281-291.

11. T. H. Lenagan and R. B. Warfield, Jr., Affiliated series and extensions of modules, J. Algebra 142 (1991), 164-187.

12. J. C. McConnell, The Nullstellensatz and Jacobson properties for rings of differential operators, J. London Math. Soc. (2) 26 (1982), 37-42.

13. J. C. McConnell and J. C. Robson, Noncommutative Noetherian rings, Wiley-Interscience, New York, 1987.

14. K. R. Pearson and W. Stephenson, $A$ skew polynomial ring over a Jacobson ring need not be a Jacobson ring, Comm. Algebra 5 (1977), 783-794.

15. J. F. Watters, Polynomial extensions of Jacobson rings, J. Algebra 36 (1975), 302-308.

Department of Mathematics, University of California, Santa Barbara, California 93106

E-mail address: goodearl 1math.ucsb.edu

Department of Mathematics, Texas A\&M University, College Station, TeXas 77843

E-mail address: letzter @math.tamu.edu 